\title{
STABILITY STUDY AND FLIGHT SIMULATION OF A BLENDED-WING-BODY UAV
}

\author{
Thomas Dimopoulos ${ }^{1}$, Pericles Panagiotou ${ }^{2}$, and Kyros Yakinthos $^{3 *}$ \\ ${ }^{1}$ Mechanical Engineer, PhD Candidate, Laboratory of Fluid Mechanics and Turbomachinery, \\ Aristotle University of Thessaloniki, 54124, Thessaloniki, Greece \\ ${ }^{2}$ Dr. Mechanical Engineer, Aristotle University of Thessaloniki, 54124, Thessaloniki, Greece \\ ${ }^{3}$ Professor in the Department of Mechanical Engineering of Aristotle University of Thessaloniki, \\ Director of the Laboratory of Fluid Mechanics and Turbomachinery, 54124, Thessaloniki, Greece
}

\begin{abstract}
This article is a product of the design process of a Blended-WingBody Unmanned Aerial Vehicle (BWB UAV). The BWB geometry blends the wing and the fuselage so that the fuselage also contributes in lift generation. This geometry reduces the lift to drag ratio significantly, however it also compromises the aircraft's stability and controllability, since there is no horizontal and vertical tail. As these features are absent from the BWB layout, the need to incorporate their functions in the new geometry arises so that they cover stability demands sufficiently, according to aircraft of similar size, use and speed. Additionally, the method used for stability studies of conventional aircraft must also be adapted. This article covers the adaptation of the method to the new BWB geometry, its results in comparison to those of conventional aircraft and the use of the results for a computational simulation of the aircraft's flight.
\end{abstract}

Keywords: UAV, BWB, stability, control, simulation

\section{INTRODUCTION}

In recent years, two of the main research directions in the field of aeronautics are related to unmanned aerial vehicles (UAVs) and to novel configurations, which deviate from the traditional tube-and-wing philosophy.

Concerning the latter, one of these alternative configurations is the Blended-Wing-Body (BWB), in which the fuselage has itself an aerodynamic shape, is smoothly connected (blended) into the wing and contributes to the generation of lift. This innovative configuration has been shown to significantly increase the lift-to-drag-ratio (L/D) of the aerial vehicle, in comparison to conventional configurations, where the fuselage produces exclusively drag. Thus, by adopting the BWB layout, significant reduction of fuel consumption, as well as increased maximum velocity with the same power plant, can be achieved. Furthermore, the BWB configuration offers increased internal volume for payload compared to a conventional configuration with similar lift generation capability.

The first essential attempt of materializing the configuration is credited to Boeing, which intended to develop a large airliner with a capacity of 450-800 passengers, reaching to the construction of the prototype X-48. Eventually, it was found that the BWB configuration cannot be employed for passenger transport in the short term, for reasons related to passenger comfort, cabin pressurization and evacuation in cases of emergency [1]. Thus, for the time

*kyak@auth.gr 
being, the BWB configuration is oriented towards other applications, including cargo transport, surveillance, research and rescue or other non-passenger-oriented missions.

All of the aforementioned missions can be accomplished with unmanned aerial vehicles (UAVs), whose mission profiles are not restricted by the limitations demonstrated by the X48 plane. The advantages of UAVs include the capability of increased autonomy (essentially limited only by fuel capacity, as the issue of commander fatigue is eliminated) and the ability to operate under harsh and/or dangerous conditions, such as in wildfires or strong winds. The combination of these advantages with the advantages of the BWB can offer new potential for a great range of missions.

However, the implementation of the BWB configuration introduces several challenges to the aircraft design procedure. This configuration, aside from the blending of the fuselage, also reduces drag by eliminating the empennage, i.e. the horizontal and vertical tail fins which govern the aircraft's stability and control. Thus, the functions of the empennage must be incorporated in the aircraft's design with a different philosophy. Moreover, as the BWB configuration constitutes a new field of research, another drawback is the lack of relevant literature and design methods to consult, whereas analytical methods of sizing and design have been established over the decades for conventional configurations of aerial vehicles

Intending to investigate the capabilities and limitations of the BWB configuration and its implementation on UAVs, the Laboratory of Fluid Mechanics and Turbomachinery (LFMT), of the Department of Mechanical Engineering at the Aristotle University of Thessaloniki, has undertaken the development, research and design of a UAV based on the BWB layout [2]. This design is integrated in the DELAER research project, coordinated by the LFMT. The project's goal is the development of an aerial platform, named DELAER RX-3 (Figure 1), providing humanitarian aid to isolated regions and islands of Greece, rapidly and at low operational cost, taking advantage of the BWB and UAV capabilities.

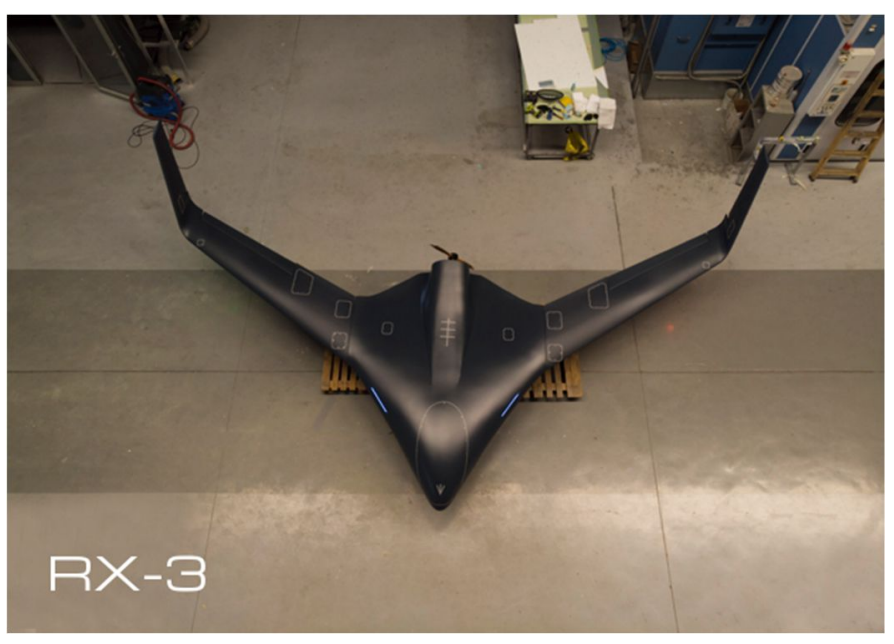

Fig. 1 The DELAER-RX-3 1:1 static mockup

The current article presents the adaptation of the conventional methodology of calculating the stability and control parameters for implementation on BWB configurations. Afterwards, the article presents the results of the modified methodology for the RX-3 and their employment in the simulation of its flight. 


\section{THEORETICAL BACKGROUND}

\subsection{Definition of the stability derivatives}

Starting from the basic kinematics theory, regarding the forces acting on the aircraft on the 3 axes of space, and based on the $2^{\text {nd }}$ Law of Newton, Equation 1 applies [3]:

$$
F_{x}=\frac{d}{d t}(m u), F_{y}=\frac{d}{d t}(m v), F_{z}=\frac{d}{d t}(m w)
$$

And similarly, regarding the moments about the 3 axes, Equation 2 applies:

$$
L=\frac{d}{d t} H_{x}, M=\frac{d}{d t} H_{y}, N=\frac{d}{d t} H_{z}
$$

Where $H_{x}, H_{y}, H_{z}$ are the angular momentum components of the body's rotation about the corresponding axis.

When Equations 1 and 2 are expanded, the forces and moments are expressed in the form of Equations 3-8:

$$
\begin{gathered}
F_{x}=m(\dot{u}+q w-r v) \\
F_{y}=m(\dot{v}+r u-p w) \\
F_{z}=m(\dot{w}+p v-q u) \\
L=I_{x x} \dot{p}-I_{x z} \dot{r}+q r\left(I_{z z}-I_{y y}\right)-I_{x z} p q \\
M=I_{y y} \dot{q}+r p\left(I_{x x}-I_{z z}\right)+I_{x z}\left(p^{2}-r^{2}\right) \\
N=-I_{x z} \dot{p}+I_{z z} \dot{r}+p q\left(I_{y y}-I_{x x}\right)+I_{x z} q r
\end{gathered}
$$

Where $u, v, w$ the linear velocity components on the axes $x, y, z$ accordingly, and $p, q, r$ the angular velocity components about the axes $x, y, z$ accordingly.

These equations are strongly nonlinear, thus it is useful to linearize them based on the small disturbance theory. According to the theory, every parameter $i$ of the problem can be expressed as the sum of a constant reference value $i_{0}$, and a disturbance $\Delta i$, sufficiently small so that the assumptions that: $\sin \Delta i \approx \Delta i, \cos \Delta i \approx 1$ and $\Delta i \cdot \Delta j \approx 0$, can be trusted.

A further simplifying assumption is symmetric initial conditions of flight and constant thrust forces, which means that $v_{o}=p_{o}=q_{o}=r_{o}=\varphi_{o}=\psi_{o}=0$. If the $\mathrm{x}$ axis for the reference condition is collinear with the aircraft's velocity, then also $w_{o}=0$.

An example of the implementation of the small disturbance theory for the simplification of motion equations is provided for the equation of forces on the $\mathrm{x}$ axis $(\mathrm{mg} \sin \theta$ is a gravityimposed term):

$$
\begin{aligned}
& X-m g \sin \theta=m(\dot{u}+q w-r v) \Rightarrow \\
& X_{o}+\Delta X-m g \sin \left(\theta_{o}+\Delta \theta\right)=m\left(\frac{d\left(u_{o}+\Delta u\right)}{d t}+\left(q_{o}+\Delta q\right)\left(w_{o}+\Delta w\right)-\left(r_{o}+\right.\right. \\
& \left.\Delta r)\left(v_{o}+\Delta v\right)\right)
\end{aligned}
$$

Considering that $v_{o}=q_{o}=r_{o}=w_{o}=0$ :

$$
X_{o}+\Delta X-m g \sin \left(\theta_{o}+\Delta \theta\right)=m \Delta \dot{u}
$$

According to the trigonometric identity

$$
\begin{gathered}
\sin \left(\theta_{o}+\Delta \theta\right)=\sin \theta_{o} \cos \Delta \theta+\sin \Delta \theta \cos \theta_{o}=\sin \theta_{o}+\Delta \theta \cos \theta_{o} \\
\Rightarrow X_{o}+\Delta X-m g\left(\sin \theta_{o}+\Delta \theta \cos \theta_{o}\right)=m \Delta \dot{u}
\end{gathered}
$$

And as the reference flight condition is governed by the equation: 


$$
\begin{gathered}
X_{o}-m g \sin \theta_{o}=0 \Rightarrow \\
\Delta X-m g \Delta \theta \cos \theta_{o}=m \Delta \dot{u}
\end{gathered}
$$

It is now assumed that $\Delta \mathrm{X}$ is a function of $\mathrm{u}, \mathrm{w}, \delta_{\mathrm{e}}$ and $\delta_{\mathrm{T}}$. Then it can be expressed as:

$$
\Delta X=\frac{\partial X}{\partial u} \Delta u+\frac{\partial X}{\partial w} \Delta w+\frac{\partial X}{\partial \delta_{e}} \Delta \delta_{e}+\frac{\partial X}{\partial \delta_{T}} \Delta \delta_{T}
$$

Where the parameters $\frac{\partial X}{\partial u}, \frac{\partial X}{\partial w}, \frac{\partial X}{\partial \delta_{e}}, \frac{\partial X}{\partial \delta_{T}}$ are the stability derivatives. The calculation of them, in their fully dimensionless form, symbolized as $C_{X_{u}}, C_{X_{w}}, C_{X_{\delta_{e}}}, C_{X_{\delta_{T}}}$, constitutes the subject of this article.

\subsection{Methodology of stability derivative calculation}

\subsubsection{Conventional methodology}

For aircraft based on conventional configurations, the calculation of stability derivatives can be conducted based on the methodology prescribed in the USAF Stability and Control DATCOM, a database of the U.S. Air Force, consolidated and codified in the practical form of a manual by Jan Roskam [4].

This methodology is largely based on empirical and experimental data, codified in charts, which take geometric and aerodynamic parameters of the aircraft as input and produce correctional coefficients for the dimensionless stability derivatives. Some of these charts may implicate up to eight geometrical parameters (rendered dimensionless in four pairs), a fact which demonstrates the method's complexity.

As already mentioned, the stability and controllability of the aircraft are governed by the empennage. Specifically, in a conventional configuration, the aircraft's rotation about the $Y$ axis (pitch) is controlled by the elevator on the horizontal tail, while rotation about the $\mathrm{Z}$ axis (yaw) is controlled by the rudder on the vertical tail. Thus, it is evident that the DATCOM method is largely dependent on the properties of the horizontal and vertical tail.

\subsubsection{Adaptation of the conventional methodology on the BWB configuration}

As the horizontal and vertical tail fins are eliminated from the RX-3, the need to incorporate their functions in the novel configuration arises, in such a way that the stability demands applying to aerial vehicles of similar size, velocity and mission profile are met. In particular:

- The control surfaces on the wing, which in a typical aircraft configuration function as ailerons, can either be deflected in counter-direction to act as ailerons, or be deflected in the same direction to act as elevators.

- The control surfaces on the winglets, positioned at an angle from the wing plane, can either apply a force on the $\mathrm{Y}$ axis to act as rudders, or apply a force on the $\mathrm{Z}$ axis to act as elevators.

The control surface layout is presented more clearly in Figure 2. 

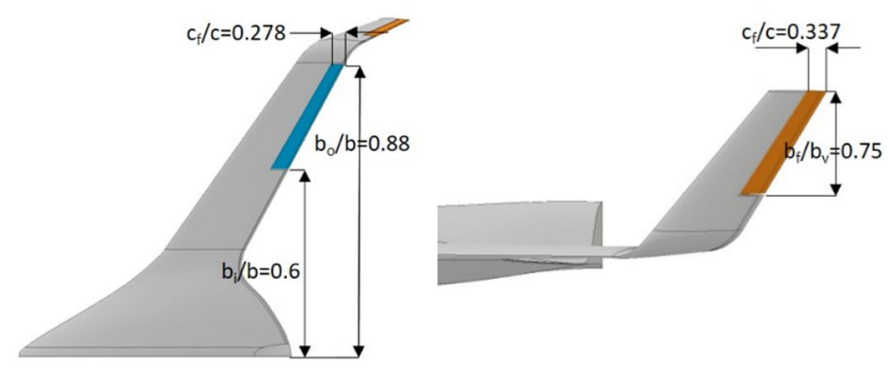

Fig. 2 RX-3 elevon (left) and ruddervator (right) layouts.

As can easily be inferred, similar modifications must be made on the implementation of the method. In particular, the modifications reported in Table 1 were made:

Table 1. Modifications of the stability derivative calculation method for implementation on the RX-3.

\begin{tabular}{|c|c|}
\hline Issue addressed & Modification applied \\
\hline Vertical tail & $\begin{array}{l}\text { As the winglets overtake the functions of the vertical tail, they } \\
\text { are treated by the methodology as a twin vertical tail. }\end{array}$ \\
\hline Horizontal tail surface & Same as the reference area of the wing, $S$. \\
\hline Elevator efficiency & $\begin{array}{l}\text { The elevators are treated with the efficiency coefficient relevant } \\
\text { to spanwise parts of the wing. It should be noted that Roskam } \\
\text { assumes a single elevator surface on the horizontal tail fin; } \\
\text { therefore, in the case of the BWB, which employs two elevator } \\
\text { control surfaces, the force and moment coefficients calculated are } \\
\text { doubled. }\end{array}$ \\
\hline $\begin{array}{l}\text { Moment arm of the } \\
\text { horizontal tail }\end{array}$ & $\begin{array}{l}\text { The distance between the aerodynamic center of the part of the } \\
\text { wing containing the elevon (due to x-axis symmetry, this distance } \\
\text { is the same for both elevons) } x_{a c_{h}} \text {, and the aerodynamic center of } \\
\text { the wing and fuselage, } x_{a c_{w f}} \text {. }\end{array}$ \\
\hline $\begin{array}{l}\text { Span } b_{h} \text { of the } \\
\text { horizontal tail }\end{array}$ & Same as that of the wing, $b$. \\
\hline $\begin{array}{l}\text { Aspect ratio } A R_{h} \text { of } \\
\text { the horizontal tail }\end{array}$ & Same as that of the wing, $A R$. \\
\hline $\begin{array}{l}\text { Lift slope of the tail } \\
\text { against the angle of } \\
\text { attack, } C_{L_{a_{h}}}\end{array}$ & Same as that of the wing, $C_{L_{a}}$. \\
\hline $\begin{array}{l}\text { Consideration of the } \\
\text { flow difference } \\
\text { between the } \\
\text { horizontal tail and the } \\
\text { wing }\end{array}$ & 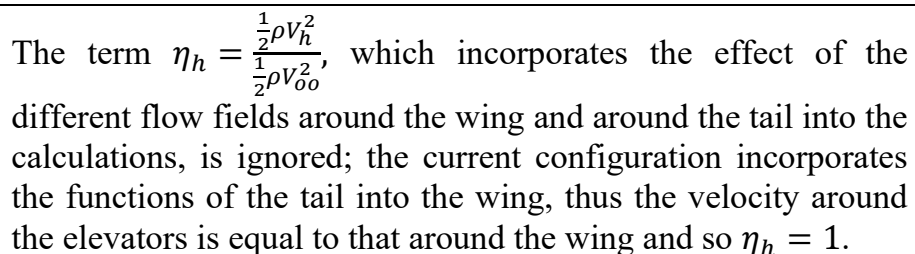 \\
\hline $\begin{array}{l}\text { Consideration of the } \\
\text { contributions of the } \\
\text { horizontal tail to } \\
\text { lateral stability terms }\end{array}$ & $\begin{array}{l}\text { Regarding the calculation of } C_{l_{\beta}} \text {, the calculation of two discrete } \\
\text { terms is implied: } C_{l_{\beta_{w f}}} \text { regarding the contribution of the } \\
\text { combination of the fuselage and wing, and } C_{l_{\beta}} \text { regarding the } \\
\text { contribution of the combination of the fuselage and tail; the two } \\
\text { terms are calculated in a similar way. Typically, this distinction }\end{array}$ \\
\hline
\end{tabular}




\begin{tabular}{|l|l|}
\hline & $\begin{array}{l}\text { is meaningful, as the horizontal acts as a secondary wing } \\
\text { regarding the rolling rotation. However, here the functions of the } \\
\text { tail are integrated in the wing and so the } C_{l} \text { term is eliminated. }\end{array}$ \\
\hline$\Delta C_{L}, \Delta C_{M}$ & $\begin{array}{l}\text { LFMT has developed an in-house low-fidelity tool, which offers } \\
\text { a rapid calculation of } \mathrm{C}_{\mathrm{L}} \text { and } \mathrm{C}_{\mathrm{m}} \text { curves for a given wing } \\
\text { configuration. The tool's philosophy is the spanwise division of } \\
\text { the wing in 2D airfoils, which are analyzed with XFLR5 [5], a } \\
\text { panel-method-based solver [6]. The results of the 2D solutions } \\
\text { are corrected to account for 3D effects, based on fundamental } \\
\text { aerodynamics theory. That way, the need for the time- and } \\
\text { resources demanding CFD computations can be kept at a } \\
\text { minimum during the conceptual and early preliminary design } \\
\text { phases, where time is of the essence. }\end{array}$ \\
\hline
\end{tabular}

\subsection{Flight Simulation}

After the calculation of the stability derivatives, a model employing automatic control methods [7], i.e. through the use the Matlab and Simulink software, is derived. This model serves to simulate the aircraft's flight and, through a flight simulation software (in particular, Flight Gear), visualize the flight. The results of this simulation can aid the designer to have a more tangible feel of the aircraft's flight behavior, before the phase of flight tests commences.

\section{RESULTS}

\subsection{DELAER-RX-3 Stability Derivatives}

The results for the most significant stability derivatives of the RX-3 are presented in comparison to the corresponding derivatives of 3 other aerial vehicles:

a. The HCUAV, which is the first UAV made by the Laboratory of Fluid Mechanics and Turbomachinery [8], Figure 3 (left).

b. The Zagi Flying Wing, a flying wing configuration UAV [9], Figure 3 (middle).

c. The Aerosonde, a conventional configuration UAV [9], Figure 3 (right).

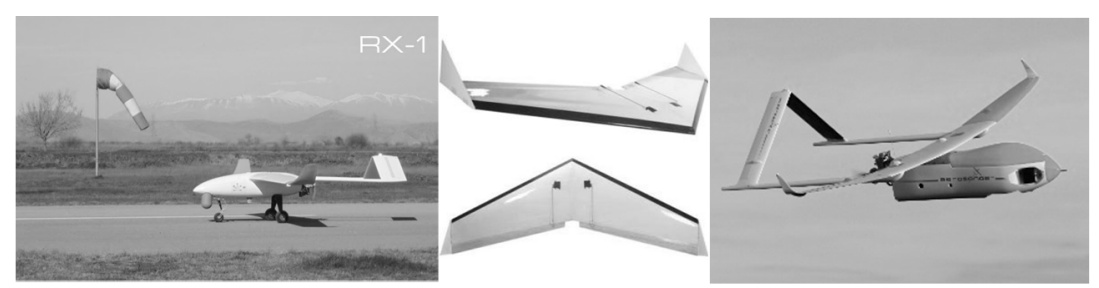

Fig. 3 (left) The HCUAV. (middle) The Zagi Flying Wing UAV. (right) The Aerosonde UAV.

This comparison constitutes a way of verifying the method, as sizes of similar size are expected to also have similar (in order of magnitude) dimensionless stability derivatives. Indeed, the results presented in Table 2 demonstrate sufficient convergence so that the method can be considered trustworthy, for preliminary design purposes. 
Table 2 DELAER-RX-3 Stability derivatives, calculated at cruise conditions (cruise velocity of $180 \mathrm{~km} / \mathrm{h}$, cruise altitude of $2000 \mathrm{~m}$ ), and comparison with stability derivatives of similar aerial vehicles.

\begin{tabular}{|c|c|c|c|c|}
\hline & DELAER & HCUAV & Zagi & Aerosonde \\
\hline$C_{L_{a}}$ & 5.91 & & 3.50 & 3.45 \\
\hline$C_{D 0}$ & 0.022 & & 0.016 & 0.03 \\
\hline$C_{m_{a}}$ & -0.70 & & -0.57 & -0.38 \\
\hline$C_{m_{\text {Selevon }}}$ & -0.57 & -0.75 & -0.32 & -0.5 \\
\hline$C_{y_{\beta}}$ & -0.51 & -0.87 & -0.074 & -0.98 \\
\hline$C_{l_{\beta}}$ & -0.11 & -0.042 & -0.028 & -0.12 \\
\hline$C_{n_{\beta}}$ & 0.087 & 0.31 & -0.0004 & 0.25 \\
\hline
\end{tabular}

The calculated stability derivatives were used for the calculation of dynamic response curves, as prescribed in [10]. The resulting curves are close to the expected.

It is noted that all stability derivatives cited are calculated at the corresponding cruise conditions, for which the assumptions of the small disturbance theory are considered safe. The flight simulation is also conducted at cruise conditions. As, during the phases of takeoff and landing, flight parameters (velocity, angle of attack, forces and moments) are intensely transitional, this analysis cannot offer sufficient information, but only a rough estimation, as stability derivatives will, too, constantly change.

The basic conditions of stability, regarding the essential moment effects about the three principal axes:

- $C_{m_{\alpha}}<0$ and $C_{m_{0}}>0$ (trim demand)

- $C_{n \beta}>0$

- $C_{l \beta}<0$

Are satisfied, except $C_{m_{0}}>0$; However, the aircraft can be trimmed with relatively small negative elevon deflection (tending to rotate the aircraft nose-up). It is thus proven that the RX-3 can sustain a steady flight. Other than that, the rest of the DELAER stability derivatives not included in Table 2, generally tend to have higher absolute values than those of the other aircraft, indicating that the DELAER is more sensitive to disturbance and applies greater forces to counteract disturbance.

\subsection{Flight Simulation}

As mentioned, after the RX-3 stability derivatives had been determined, they were utilized as input in a model simulating the flight of the aircraft. This model was made with the help of Simulink, which organizes all equations in the form of a flowchart, demonstrating the flow from input data to the output it produces.

The model has a high degree of complexity and many feedback loops, elements which reflect the amount of information input, as well as the multitude of equations required for the calculation of all flight parameters.

The execution of the simulation demonstrated a smooth flight behavior, as prescribed by established flight standards, and was verified against a similar tailless configuration [11].

It should be noted that even this simulation follows a simplified approach and can under no conditions diminish the significance of flight tests. For instance, parameters such as the randomness of environmental conditions and fluctuations in air density are ignored. 
However, the described methodology offers a first test of the aircraft's airworthiness. The execution of determined maneuvers and measurement of the aircraft's response to them, give the RX-3 designers a tangible feel of the characteristics of flight stability and performance, as well as guidelines for potential improvement.

\section{CONCLUSIONS}

From the results presented, it is demonstrated that the semi-empirical method can approach the properties of an unconventional configuration under relatively few modifications. The accuracy of this modified methodology can be verified with CFD runs and test flights.

The comparison of the RX-3 stability parameters with the corresponding parameters of other aerial vehicles shows similar magnitudes, which proves that a BWB configuration aircraft can be controlled and navigated equally well as a conventional configuration aircraft. This satisfactory accordance between results may also be interpreted as an indication, albeit not concrete proof, of the accuracy of the computational tool.

The same conclusions are inferred by the visualization of the flight with flight simulator software.

The project was materialized under the framework RESEARCH - CREATE- INNOVATE and cofunded by the European Union and Greek national funds through the O.F. Competitiveness, Entrepreneurship and Innovation (ЕПАvEK) (project code: T1E $\Delta \mathrm{K}-01262)$.

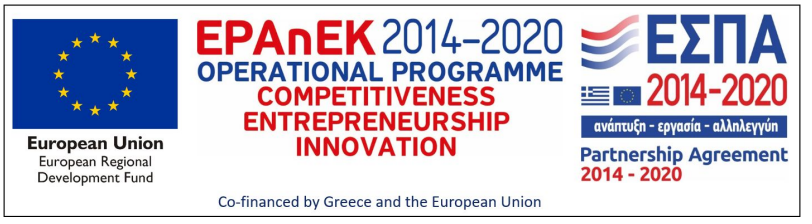

\section{REFERENCES}

1. R.H. Liebeck, J AIRCRAFT 41, 10-25 (2004).

2. P. Panagiotou, K. Yakinthos, Parametric aerodynamic study of Blended-Wing-Body platforms at low subsonic speeds for UAV applications, Denver, Colorado: AIAA Aviation Forum, 2017.

3. R.C. Nelson, Flight Stability And Automatic Control, McGraw-Hill, 1989.

4. J. Roskam, Airplane Design, Part VI: Preliminary Calculation of Aerodynamic, Thrust And Power Characteristics, University Of Kansas, 1987.

5. A. Deperrois, XFLR5: Analysis of foils and wings operating at low Reynolds number. 2013.

6. J.D. Anderson, Fundamentals of Aerodynamics. Tziola (Greek edition) (2016).

7. R.C. Dorf, R.H. Bishop, Modern Control Systems, Tziola (Greek edition) (2016).

8. P. Panagiotou, P. Kaparos, C. Salpingidou, K. Yakinthos, AEROSP SCI TECHNOL 50, 127-138 (2016).

9. R.W. Beard, Small Unmanned Aircraft (Theory And Practice), Princeton University Press, 2012.

10. J. Roskam, Airplane Design, Part VII: Determination of Stability, Control and Performance Characteristics: FAR and Military Requirements. University Of Kansas, 1988.

11. Gary L. Teper, Aircraft Stability and Control Data, NASA CR-96008, 1969 